\title{
A NOVEL TECHNIQUE FOR THE DIRECT DETERMINATION OF CARRIER DIFFUSION LENGTHS IN GaAs/AIGaAs HETEROSTRUCTURES USING CATHODOLUMINESCENCE
}

\author{
P. C. Sercel, H. A. Zarem, J. A. Lebens, L. E. Eng, A. Yariv, and K. J. Vahala \\ Department of Applied Physics, 128-95 \\ California Institute of Technology \\ Pasadena, California 91125 \\ $818 / 356-2194$
}

\begin{abstract}
A new technique for determining carrier diffusion lengths in direct gap semiconductors by cathodoluminescence measurement is presented. Ambipolar diffusion lengths are determined for GaAs quantum well material, bulk GaAs, and $\mathrm{Al}_{x} \mathrm{Ga}_{1-x} \mathrm{As}$ with $\mathrm{x}$ up to 0.38. A large increase in the diffusion length is found as $x$ approaches 0.38 and is attributed to an order of magnitude increase in lifetime.
\end{abstract}

\section{INTRODUCTION}

Measurement of material parameters such as carrier diffusion length in $\mathrm{GaAs} / \mathrm{AlGaAs}$ heterostructures is of great interest due to the importance of these structures for optoelectronic devices, high electron mobility transistors (HEMT), resonant tunneling diodes, etc. Minority and ambipolar carrier diffusion lengths are important both as transport parameters and as quantities which can be used to obtain information on carrier lifetime or mobility. Many methods have been used to measure diffusion lengths, most notably, the HaynesShockley experiment [1]. More recently, techniques such as short-circuit photocurrent measurements [2], electron beam induced currents [3], transient grating techniques [4], and time of flight studies [5] have been applied. Here, we present a simple new technique for determining the carrier diffusion length using cathodoluminescence (CL). This method can be applied to direct gap semiconductors to determine either minority carrier or ambipolar diffusion depending on the sample doping and excitation conditions. Due to the precision and ease with which one can control the position of the electron beam in a scanning electron microscope (SEM) the method is very straightforward and accurate. Furthermore, the ability to examine the sample under the high magnifications available with an SEM allows this technique to be applied to submicron devices and arrays of microstructures such as quantum wires. In this paper we apply the technique to ambipolar diffusion in GaAs quantum well material,

bulk $\mathrm{GaAs}$, and $\mathrm{Al}_{x} \mathrm{Ga}_{1-x} \mathrm{As}$ with aluminum mole fraction $\mathrm{x}$ up to 0.38 . Transport in the $\mathrm{X}$ and $\mathrm{L}$ valleys is found to significantly affect the diffusion length in higher aluminum material.

\section{DESCRIPTION OF THE TECHNIQUE}

The experiment is performed in a modified SEM with a fiber optic CL collection system [6]. In the SEM, an energetic electron beam is incident upon the sample, generating electron hole pairs within the interaction region of the beam with the sample. One end of the fiber is placed approximately $0.5 \mathrm{~mm}$ from the sample, collecting luminescence from a region approximately $100 \mu \mathrm{m}$ in radius while the other end is coupled into a monochrometer allowing spectral resolution of the CL signal. A thin aluminum mask covers half of the sample and prevents detection of the luminescence emanating from the region under it. All detected luminescence is from radiative recombination which occurred in the unmasked region. This is shown schematically in Fig.1. By generating the carriers in the masked region of the sample and measuring the luminescence intensity as a function of the distance of the beam from the mask edge, $\mathrm{I}(\mathrm{x})$, we are able to determine the diffusion length, $\mathrm{L}_{\mathrm{D}}$. To allow different material compositions to be investigated on a single sample, carriers were confined to narrow channels of the material being studied by high bandgap barriers on either side. It is important that the barriers be thin compared to $\mathrm{x}$ so that only those carriers which were generated in the channel contribute to its CL signal. Signals from different channels were separated with the monochrometer.

The simplicity of the technique results from the fact that the luminescence signal depends exponentially on $x / L_{D}$. Because of the large collection area of the optical fiber, the CL signal is proportional to the net flux of carriers into the unmasked region. Equation 1 governs the concentration of carriers outside of the generation region

$$
0=-\frac{\mathrm{C}}{\tau}+\mathrm{D} \nabla^{2} \mathrm{C}
$$

where C, $\tau$ and D are the carrier concentration, lifetime, and diffusivity. In the one dimensional problem, the solution to Eq. 1 is simply $C(x)=C_{0} \exp \left(-x / L_{D}\right)$, where $L_{D}=\sqrt{D \tau}$. The corresponding flux is also proportional to $\exp \left(-x / L_{D}\right)$. In addition, this solution would apply if we generated a line of constant carrier density, parallel to the mask edge. By breaking the line into infinitesimal elements, and examining the flux due to an individual element, it can be shown that the net flux caused by a single point source is also proportional to $\exp \left(-x / L_{D}\right)[7]$. This argument is easily extended to an arbitrary generation region. The exponential dependence

11.3.1 
on $x$ is significant not only because of the ease with which it is handled numerically, but also because it allows us to ignore the size and shape of the interaction region so long as it remains completely under the aluminum mask.

\section{DESCRIPTION OF THE EXPERIMEN'}

The samples used in this experiment were grown by molecular beam epitaxy in a Riber $2300 \mathrm{R} \& \mathrm{D}$ system. Layers with $\mathrm{Al}$ mole fractions of $0.09,0.19,0.25,0.35$, and 0.38 were grown as well as a GaAs layer and a $100 \AA \mathrm{GaAs}$ quantum well. There was no intentional doping in any of the samples. The determination of the $\mathrm{Al}$ mole fraction was accomplished by room temperature photoluminescence (PL) measurements. The PL spectra were taken using a relatively low intensity $\left(\approx 1 \mathrm{~W} / \mathrm{cm}^{2}\right)$ excitation by the $5145 \AA$ line of an Argon ion laser, and $\mathrm{Al}$ mole fractions, $x$, were determined from the PL spectra peak energy, $E_{\mathrm{p}}$, using the relation $E_{p}(\mathrm{eV})=1.42+1.45 x-0.25 x^{2}$ which has been shown to hold for $0<x \leq 0.45$ and similar excitation conditions [8]. There is some disagreement as to the relationship between bandgap energy and $\mathrm{Al}$ mole fraction $[9,10]$, particularly in the range $0.35<x<0.45$. We have used the relationship of Ref. [9] since it is more recent.

To form the mask, stripes of aluminum, $750 \AA$ thick and $100 \mu \mathrm{m}$ wide were evaporated onto the surface of the samples. While thin enough to allow the electron beam to penetrate into the sample with minimal scattering, this mask was found to be sufficiently thick to prevent detection of a luminescence signal through it. All data presented here were taken at room temperature.

The sample was excited by a $10 \mathrm{kV}$ electron beam with a current of approximately $20 \mathrm{nA}$. This yields a carrier density on the order of $10^{18} \mathrm{~cm}^{-3}$ in the generation volume. With a $10 \mathrm{kV}$ beam, the generation volume has a radius of approximately $0.1 \mu$ in the channels [11]. To ensure that the entire interaction region remained under the mask scans were performed over distances greater than $0.9 \mu$ from the mask edge. As an additional precaution the beam was scanned toward the mask edge to ensure that material between the beam and the mask edge had not been altered by the beam. Figure 2 shows typical data runs for three different channels.

\section{INTERPRETATION OF THE DATA}

Figure 3 shows the results of the diffusion length measurements. The diffusion lengths given represent an average over four measurements in different regions of the sample. The error bars represent the standard deviations. For the quantum well material, $L_{D}$ is approximately the same as in the $\mathrm{GaAs}$ channel. The reduction in the diffusion length for the $\mathrm{Al}_{.19} \mathrm{Ga}_{.81}$ As channel can be attributed to a reduced mobility due to alloy scattering and increased effective mass [12]. A surprising result is the trend towards extremely large diffusion lengths for the $\mathrm{Al}_{x} \mathrm{Ga}_{1-x} \mathrm{As}$ material for $\mathrm{x}$ beyond 0.2 . Increased alloy scattering, intervalley scattering, and effective mass, cause further reductions in the mobility leading us to conclude that it is an increased lifetime which is respon-

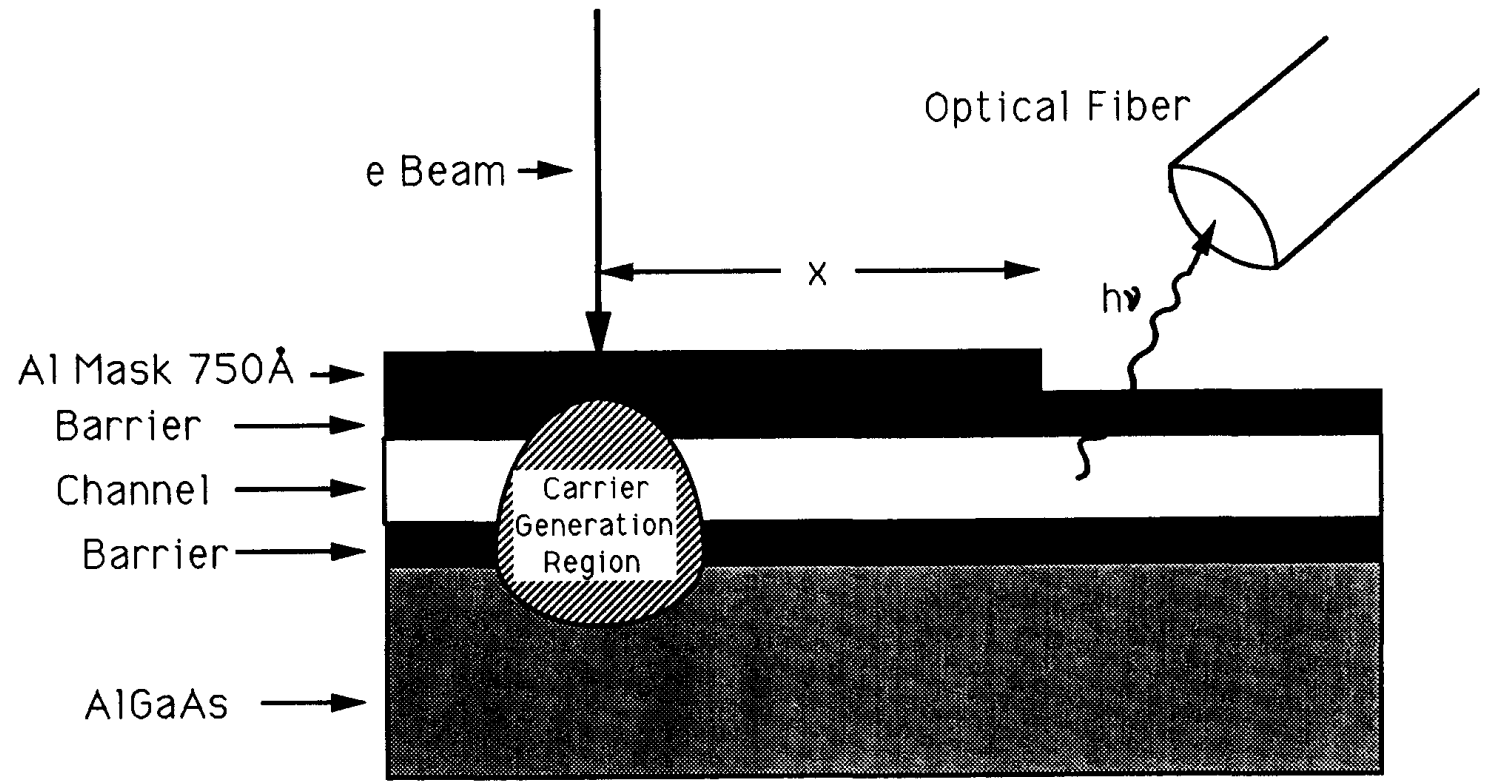

Figure 1: Schematic diagram of the experiment. An electron beam is incident on the sample, generating electron hole pairs. Luminescence is collected with the optical fiber. The $\mathrm{Al}$ mask blocks detection of luminescence from the region under it. 
sible for the large values of $L_{D}$. Using the electron mobility data of Ref. [12], and a heavy hole mobility calculated from Ref. [13], our $L_{\mathcal{D}}$ measurements imply an order of magnitude increase in lifetime for the 0.35 and $0.38 \mathrm{Al}$ mole fraction samples as compared to GaAs.

We believe the increase in lifetime to be due to an increase in the occupancy of the indirect $X$ and $L$ valleys as the aluminum mole fraction is increased towards 0.4 , atwhich point AlGaAs becomes an indirect gap semiconductor. As the $\mathrm{Al}$ mole fraction is increased from zero to 0.35 , the energy separation between the $\Gamma, X$, and $L$ valleys decreases to less than $0.05 \mathrm{eV}[10,9]$. This situation persists over the range $0.35<x<0.45$. In this region, all three valleys will be significantly populated under room temperature excitation. Since intervalley scattering and intraband relaxation processes all occur on a time scale which is much shorter than the carrier lifetime $[14,15]$, the carriers in the conduction band may be assumed to be in thermal equilibrium, and Fermi-Dirac statistics may be applied. We have calculated the populations of the three valleys of the conduction band using Fermi-Dirac statistics and the parabolic band approximation. The bandgaps for these valleys were taken from Ref. [9] and the density-of-states effective masses from Ref. [13]. We define the parameter $R(n) \equiv n / n_{\Gamma}$ where $n$ is the carrier concentration and $n_{\Gamma}$ is the population of the $\Gamma$ valley. On average, each electron will spend $1 / R$ of its life

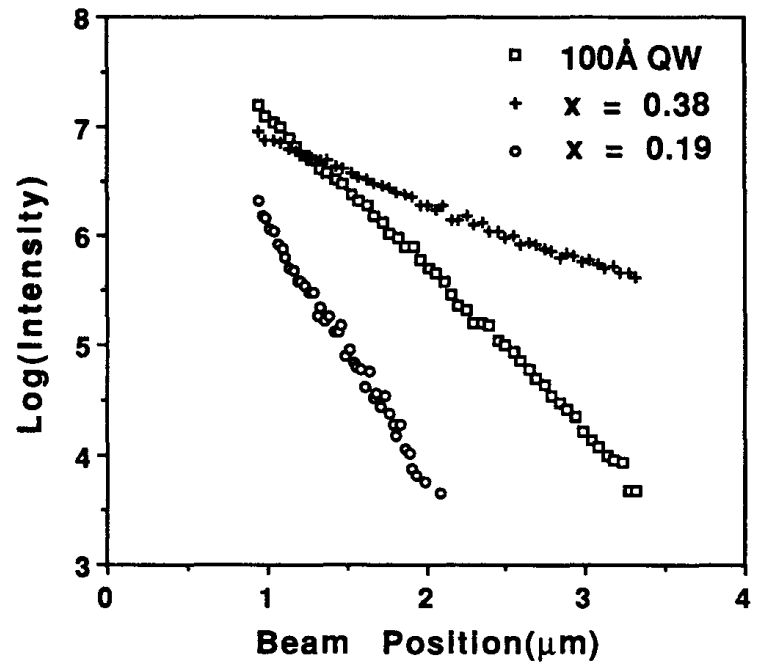

Figure 2: $\log$ of the cathodoluminescence intensity as a function of beam position for quantum well, $\mathrm{Al}_{38} \mathrm{Ga}_{.62} \mathrm{As}$ and $\mathrm{Al}_{19} \mathrm{Ga}_{.81} \mathrm{As}$ channel. The ambipolar diffusion length is given by the reciprical of the slope. in the $\Gamma$ valley. Taking the lifetime in the indirect valleys as infinite compared to the lifetime in the $\Gamma$ valley, $R$ can be viewed as a lifetime enhancement factor, that is, the carrier lifetime is given by $\tau=R \pi_{\Gamma}$, where $\tau_{\Gamma}$ is the lifetime in the $\Gamma$ valley. Using an approximate value for the carrier concentration of $10^{18} \mathrm{~cm}^{-3}$ we find for $\mathrm{Al}_{38} \mathrm{Ga}_{.62} \mathrm{As} R \approx 10$ in agreement with our expectation. To obtain a quantitative confirmation of our hypothesis would require precise knowledge of the carrier concentrations, or a direct measurement of carrier lifetime as a function of aluminum mole fraction. Further work to measure these lifetimes is in progress.

In conclusion, we have presented a new method for measuring either minority carrier or ambipolar diffusion lengths by cathodoluminescence. The technique is extremely accurate due to the precision with which one can position an electron beam in an SEM. A simple exponential dependence of the CL intensity with beam position facilitates extraction of $\mathrm{L}_{\mathrm{D}}$ from the data. A trend towards large $\mathrm{L}_{\mathrm{D}}$ was observed for $\mathrm{Al}_{x} \mathrm{Ga}_{1-x} \mathrm{As}$ with $\mathrm{x}$ approaching 0.38 and was explained to result from intervalley scattering. Further investigations of this effect are in progress.

The authors would like to acknowledge the support of the Office of Naval research and the SDIO-ISTC. One of us (P.S.) would like to acknowledge the support of a graduate NSF fellowship.

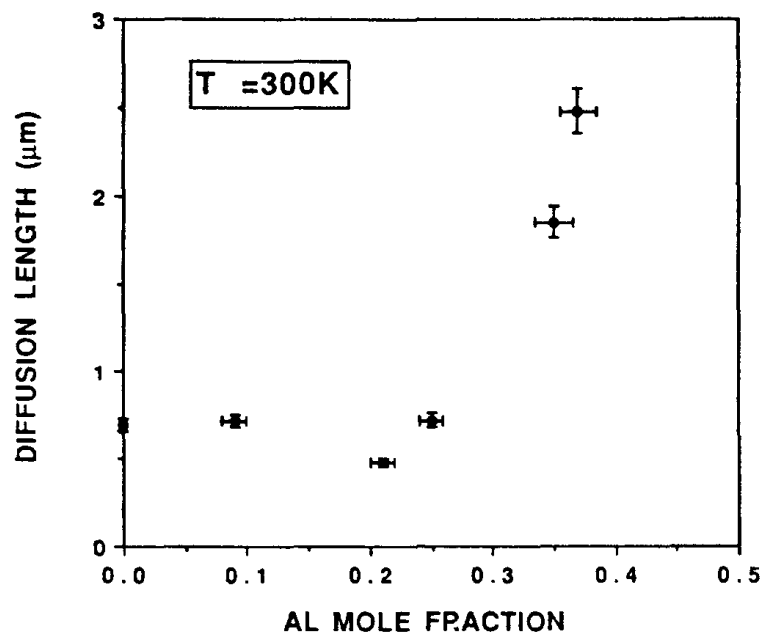

Figure 3: Ambipolar diffusion length at a carrier density of $10^{17}-10^{18} \mathrm{~cm}^{-3}$. The samples with $\mathrm{Al}$ mole fraction greater than 0.3 have high values due to the increased lifetime. A $100 \AA \mathrm{GaAs}$ quantum well showed the same value as the thicker GaAs $(x=0.0)$ layer. 


\section{References}

[1] J. R. Haynes and W. Shockley,Phys. Rev., 81, 835 (1951).

[2] H. C. Casey Jr., B. I. Miller, and E. Pinkas, J. Appl. Phys.,44, 1281 (1973).

[3] C. van Opdorp, R. C. Peters, and M. Klerk, Appl. Phys. Letts., 24, 125 (1974).

[4] K. Hattori, T. Mori, H. Okamoto, and Y. Hamakawa, Appl. Phys. Letts., 51 (1987).

[5] H. Hillmer, S. Hansmann, A. Forchel, E. Lopez, H. P. Meier, and K. Ploog, Appl. Phys. Letts., 53 (1988).

[6] M. E. Hoenk and K. J. Vahala, Rev. Sci. Instrum., 60, 226 (1989).

[7] W. Van Roosbroeck, J. Appl. Phys., 26, 380 (1955).

[8] N. C. Miller, S. Zemon, G. P. Werber, and W. Powaazinik, J. Appl. Phys., 57, 512 (1985).

[9] H. J. Lee, L. Y. Juravel, J. C. Woolley, and A. J. SpringThorpe Phys. Rev. B, 21, 659 (1980).

[10] H. C. Casey, Jr. and M. B. Panish, Heterostructure Lasers (Academic Press, New York, 1978).

[11] H. J. Leamy, J. Appl. Phys., 53, R51 (1982).

[12] A. K. Saxena, Phys. Rev. B, 24, 3295 (1981).

[13] S. Adachi, J. Appl. Phys., 58, R1 (1985).

[14] C. L. Tang and D. J. Erskine, Phys. Rev. Letts, 51, 840 (1983).

[15] J. Shah, A. Pinczuk, A. C. Gossard, and W. Wiegmann, Phys. Rev. Letts., 54, 2045 (1985). 